\title{
Theoretical Study of Line and Terraces Defects on GaN(0001) Surface
}

\author{
Xiaoqin Liang ${ }^{1, a}$, Qiu Lifen ${ }^{2, b}$,Ping Huang ${ }^{2, c}$, Chun Yang ${ }^{1, d^{*}}$ \\ ${ }^{1}$ College of Chemistry and Material Science, Sichuan Normal University, Chengdu 610068, China \\ ${ }^{2}$ College of Physics and Electronic Engineering, Sichuan Normal University, Chengdu 610068, \\ China
}

alxqygr@163.com, ${ }^{b} 714732432 @ q q . c o m,{ }^{c} 123023237 @ q q . c o m,{ }^{d}$ chuanyang_2000@263.net

Keywords: GaN (0001) surface, line defects, terrace, DFT

Abstract. a vacancy line defects on the $\left[{ }^{112} 0\right]$ and $[10 \overline{1} 0]$ direction; the GaN single-layer terraces defects of $\mathrm{GaN}(0001)$ surfaces are studied by first-principle calculations based on density functional theory. The surface energy, charge density, and Mulliken charge population of defect surfaces are calculated, and the surface geometries of before and after optimization for clean and defect surfaces are compared. Upon completion of the result calculation, it was revealed that the surface atoms of the line defect on the $[10 \overline{1} 0]$ direction actually relax along the same direction. The stability of defect surface in the $\left[{ }^{10} \overline{1} 0\right]$ direction is better than that in the $[11 \overline{2} 0]$ direction. The terraces surface on the $[1 \overline{2} 0]$ direction is the most stable, which is consistent with the experimental observation. Terraces surface platform on the $[11 \overline{2} 0]$ and $[10 \overline{1} 0]$ directions are respectively inclination $0.7^{\circ}$ and $0.2^{\circ}$ towards the $\left[1^{1} 00\right]$ and $\left[1^{2} 10\right]$ direction. Terrace defects can enhance the surface metallicity, which is helpful for the adsorption of exotic particles.

\section{Introduction}

In the last decade, GaN has been investigated intensively, both in experiment and in theory ${ }^{[1-4]}$. The of GaN's wide band gap has captured the attention of scholars due to its promising potential in optoelectronics, and high-speed and high-power devices ${ }^{[5-6]}$. III-V semiconductors such as GaN have direct band gap and higher saturated electron mobility in comparison to Si. Due to the unique qualities of perovskite oxides ${ }^{[7-8]}$ allowing their fusion with $\mathrm{GaN}$ based integrated circuits for futuristic multipurpose electronics, many efforts have been devoted to the growth of oxide thin films on GaN. GaN has been the most intensively studied material among the group-III nitrides by far. The structural and electronic properties of GaN surfaces depend sensitively on the orientation of the surface, surface termination, and reconstruction ${ }^{[1-4]}$. The most common growth direction of epitaxial hexagonal GaN is normal to the 0001 basal plane ${ }^{[9]}$. However, the surface of GaN film growth on heterogeneous substrate usually contains all kinds of common defect types, such as point, line and areal defects. All of these defects can have either a direct or indirect influence on the quality of integration of ferroelectric thin films.

During the experiment, the surface defects of $\mathrm{GaN}$ were reported, stating that William ${ }^{[10]}$ observed three kinds of defect structures on $\mathrm{GaN}$ (0001) surfaces by scanning tunneling microscopy: (i) striations spaced $\sim 50 \AA$ apart, running perpendicular to the step edges; (ii) row defects running at an angle of $30^{\circ}$ with respect to the step edges and separated by $\sim 16 \AA$; and (iii) oval defects about ( 8 $\AA) \quad(16 \AA)$ in size. striations terraces on the direction $[1 \overline{2} 10] \mathrm{GaN}$ (0001) surface growth in rich nitrogen have been reported ${ }^{[11]}$. Shen ${ }^{[12]}$ investigated single and multi-layer terraces by using an atomic force microscope, including striation on [11 $\overline{2} 0]$ direction and " $\mathrm{z}$ " type terraces on [10 $\overline{1} 0]$ and $[01 \overline{1} 0]$ direction respectively. The single atomic terrace and dislocation defects on the GaN (0001) surface were confirmed simultaneously by Heiji ${ }^{[13]}$, while experimental results also show that epitaxy growth of STO film on GaN-substrate is not along the epitaxy orientation of the lesser crystal lattice mismatch: (111)SrTiO3//(0001)GaN, [1 $\left.{ }^{\overline{1}} 0\right] \mathrm{SrTiO} 3 / /\left[100^{\overline{1}} 0\right] / / \mathrm{GaN}$, but [1 $\left.{ }^{\overline{1}} 0\right] \mathrm{SrTiO} / /\left[11^{\overline{2}} 0\right]$ 
orientation along $\mathrm{c}$ axis rotation $30^{\circ}$ on $\left[{ }^{11 \overline{2} 0}\right]$ and $\left[{ }^{10} \overline{1} 0\right]$ direction, which is potentially relate to these direction defects.

In the present experimental study, the form of $\mathrm{GaN}$ defects surface is only got, and the atomic relaxation near defects is still unclear. For the surface defect is how to influence the surface properties and stability, no research and related explanation have been given. Theoretical studies on GaN surface have a low concentration, those that have been conducted are related to volume defect and clean surface $^{[14-16]}$. Our group ${ }^{[17]}$ has discussed six kinds of point defect models. During that time, migrate rate, bonding, atomic relaxation and the formation energy of eight kinds of point defects on $\mathrm{GaN}(0001)$ surface were calculated ${ }^{[18]}$. It is worth mentioning that theoretical simulation of line, terrace and mixed defects are hardly studied and reported.

Until recently, the microscopic details of defect forms are unclear. There is very little know about rebonding and the reconstruction of the surfaces, and even after reviewing the experimental data it can be difficult to determine the location and cause of the reaction. In this paper, first-principle calculations have provided valuable information about defect surface, including geometrical structure, atomic population, charge density of atoms near defects, and emphasizes surface defect effects on surface properties and stabilities. It is worth mentioning that theoretical simulation of line, terrace and mixed defects are hardly studied and reported.

\section{Computation models and method}

To assist with the experiment, GaN bulk super cells containing twelve cells each are fashioned as reference models. Ga-terminated GaN(0001) slab surface models are then obtained after slicing, in which vacuum layer height is defined as $16 \AA$ at $\mathrm{Z}$ axis positive direction $((0001)$ direction), and the surface at $\mathrm{Z}$ axis negative direction has been saturated by the pseudo-hydrogen. Therefore, these findings prove that cyclic enantimorphous interaction between the upper and lower surface is able to be effectively prevented. Throughout this work, line defect models in the direction of $\left[1{ }^{1 / 2} 0\right]$ and $[10 \overline{1} 0]$ on the $\mathrm{GaN}(0001)$ surface are designed and studied as straight terraces with all step heights of $2.590 \AA$ ( one Ga-N bilayer high), in which terrace widths are respectively $3.659 \AA$ and $1.584 \AA$ in the $\left[{ }^{11 \overline{2} 0}\right]$ and $[10 \overline{1} 0]$ direction on the $\mathrm{GaN}(0001)$ surface.
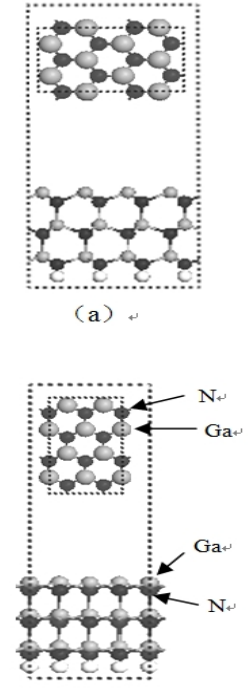

(d)
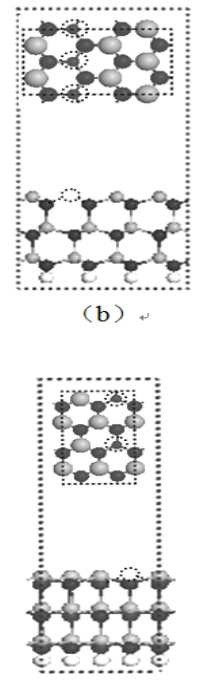

(e)

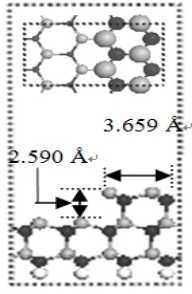

(c)

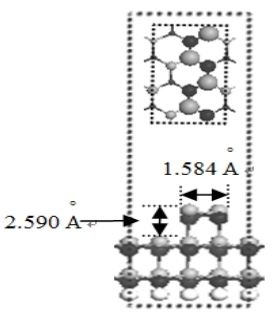

(f)

Fig. 1 lists the view of $\mathrm{GaN}$ from the side and vertical viewpoints, in which the vertical views are located at the top of each image, while the side views are located at the bottom. Images (a) and (d)

respectively indicate the $\left[{ }^{11 \overline{2} 0}\right]$ and $\left[{ }^{10 \overline{1}} 0\right]$ direction of the clean surface; while (b) and (c) respectively indicate line and terrace defectsurface in the $\left[{ }^{1120}\right]$ direction. Finally, (e) and (f) display line and terrace defect surface in the $\left[{ }^{10 \overline{1}} 0\right]$ direction. The dotted line around the perimeter of each image denotes vacancy $\mathrm{Ga}$ atoms. 
The calculations are performed by density functional theory (DFT) with the generalized gradient approximation (GGA). Perdew-Wang91 is chosen as the exchange and correlation function, and the spin-polarization effects are considered. Firstly, the lower precision (Ecut=260eV) is selected. Then, the base higher precision $($ Ecut $=300 \mathrm{eV})$ is selected to optimize the models, and the energies of final optimized models are calculated. Pseudo-potential approach is used to describe interaction potential, the $3 \mathrm{~d} 104 \mathrm{~s} 24 \mathrm{p} 1$ of $\mathrm{Ga}$ atoms and $2 \mathrm{~s} 22 \mathrm{p} 3$ of $\mathrm{N}$ atoms are treated as valence electrons. SCF convergence criterion is set to $2.0 \times 10-6 \mathrm{eV} /$ atom. A $3 \times 2 \times 1$ Monkhorst-Pack mesh is used to sample the $\mathrm{K}$ point of Brillouin zone. The bottom three layers of atoms (containing $\mathrm{H}$ atom) are fixed in order to reduce calculation time. Throughout the experiment, all calculations are performed using CASTEP simulation package.

\section{Results and discussion}

Geometry structure analysis: Generally speaking, atoms on the clean surface are not reconstruction but relaxation. Clean $\mathrm{GaN}(0001)$ surface has been optimized in order to compare with defect surface, and table 1 gives the corresponding data of atomic relaxation on GaN surface. In table $1 \Delta \mathrm{z}$ is defined as the difference of before and after relaxation for line defect and clean surface; while "+" and "-" indicate respectively that the distance increase and decrease. The left and right sides of " " respectively indicate minimum and maximum, or its percentage difference of atomic space of the same adjacent layer after relaxation. Our calculation results are essentially consistent with literature value ${ }^{[19]}$. According to table 1, the atomic relaxation extent ranges from smallest at the inside to largest at the outside. The forth layer atoms only relax $0.026 \AA$ toward inside, surface existence has little effect on relaxation for the fifth layer atom. Atomic space between surface layer and the next surface layer expands about $13.1 \%$, which is rather large comparing with literature value $10.8 \%$. Atomic space between the second and the third surface layer expands about $0.3 \%$, whereas literature value is $3.6 \%$. It is likely that this is caused because the surface mentioned by the literature has been restructured, and has a large deviation regarding lump materials. However, the calculated value found in this paper is close to inner parameter. The atomic space existing firstly between the third and the fourth layer, and secondly between the fourth and the fifth layer, expand respectively at $15.7 \%$ and $-1.3 \%$, obvious change of the former is as a result of atoms of the third layer and the forth layer respectively relax to outside and inside.

Table 1 Atomic space before and after relaxation for clean surface and line defect surface of $\operatorname{GaN}(0001)(\AA)$

\begin{tabular}{|c|c|c|c|c|c|c|}
\hline & \multicolumn{2}{|c|}{ Clean surface } & \multicolumn{2}{|c|}{$\begin{array}{c}{[11 \overline{2} 0] \text { line defect }} \\
\text { after relaxation }\end{array}$} & \multicolumn{2}{|c|}{$\begin{array}{c}{[10 \overline{1} 0] \text { Line defect }} \\
\text { after relaxation }\end{array}$} \\
\hline & $\begin{array}{c}\text { Before } \\
\text { relaxation }\end{array}$ & $\begin{array}{c}\text { After } \\
\text { relaxation }\end{array}$ & Atomic space & $\Delta \mathrm{z} / \%$ & $\begin{array}{l}\text { Atomic } \\
\text { space }\end{array}$ & $\Delta \mathrm{z} / \%$ \\
\hline $\mathrm{z}_{12}$ & 0.649 & $0.734[0.72]$ & $0.304 / 0.787$ & $-58.6 / 7.2$ & $0.383 / 0.383$ & $-47.8 /-47.8$ \\
\hline $\mathrm{z}_{23}$ & 1.944 & $\begin{array}{c}1.938[2.01] \\
{[19]}\end{array}$ & $1.871 / 2.026$ & $-3.5 / 4.5$ & $1.935 / 2.023$ & $0.2 / 4.4$ \\
\hline $\mathrm{Z}_{34}$ & 0.649 & 0.751 & $0.704 / 0.863$ & $-6.3 / 14.9$ & $0.763 / 0.792$ & $1.6 / 5.5$ \\
\hline $\mathrm{Z}_{45}$ & 1.944 & 1.919 & $1.901 / 1.935$ & $-0.9 / 0.8$ & $1.924 / 1.937$ & $0.3 / 0.9$ \\
\hline
\end{tabular}

Geometry's side view of $\mathrm{GaN}(0001)$ line defect surface after relaxation is showed in Fig.

2. As seen Fig.2, effects of two kinds of defects are relative large on surface atoms, and on under surface atoms is relatively small (table 1). When compared with a clean optimized su 


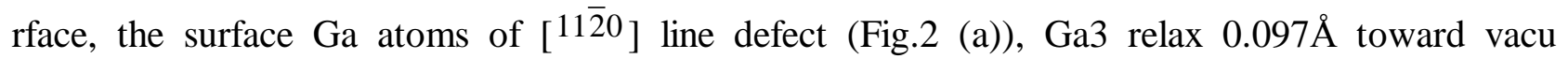
um layer, Ga1 and $\mathrm{Ga} 2$ relax respectively $0.416 \AA$ and $0.430 \AA$ toward inside. The distance $\mathrm{b}$ etween the $\mathrm{Ga} 3$ and $\left[{ }^{11 \overline{2}} 0\right]$ line defect has maximum in consideration of the periodicity of $\mathrm{cr}$ ystals. So that $\left[{ }^{11 \overline{2} 0}\right]$ line defect effect on surface atoms decreases along with the increase o $\mathrm{f}$ the distance. For a clean, unoptimized surface, $\mathrm{Ga} 1, \mathrm{Ga} 2$ and $\mathrm{Ga} 3$ relax respectively 0.533, -0.014 and $-0.000 \AA$ and the position of surface $\mathrm{Ga}$ atom near defect after relaxation is go od agreement with no relaxation atoms for clean surface. The atomic relaxations found benea th the initial layer at the object's surface are divided into two respective components: space between adjacent layer atoms along $\left[{ }^{1} 100\right]$ direction contracts, whereas space expands along [1100] direction in Fig.2, in which the line defect (which is drawn as a dotted line) is conv eyed as the reference center. Relaxation of $\mathrm{Ga}$ atoms on surface relax towards the inside eq ually, $0.378 \AA$, and space between the first layer and the second layer atoms contracts $47.8 \%$ (table 2), which is similar with the surface containing [10 $\overline{1} 0]$ line defect. Relative to [11 $\overline{2} 0$ ] line defect surface, atomic relaxations of the $[10 \overline{1} 0]$ line defect surface are proven to have a greater order, and tend to have very little effect on their surface's atomic relaxation.

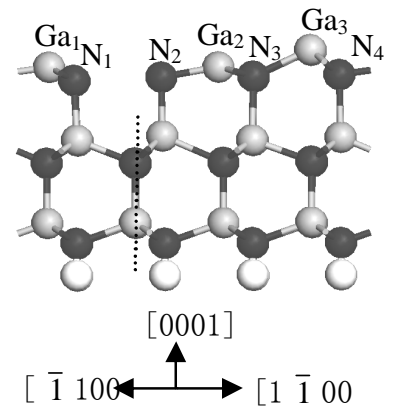

(a) Surface of $[11 \overline{2} 0]$ line defect

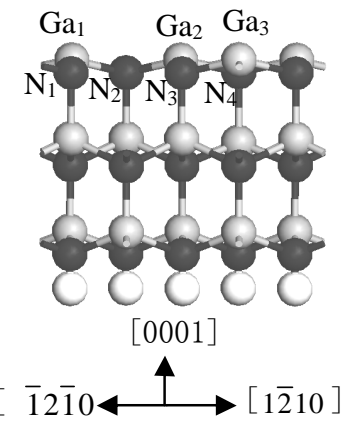

(b) Surface of [ $10 \overline{1} 0]$ line defect

Fig. 2 Side views of optimized geometry for $\mathrm{GaN}(0001)$ line defect surface.

Fig. 3 and Fig. 4 give respectively optimized geometry of [ $\left[1{ }^{110}\right]$ and $[10 \overline{1} 0]$ terrace on $\mathrm{GaN}(0001)$ surface. The same height changes are observed for surface $\mathrm{Ga}$ atoms and the second layer $\mathrm{N}$ atoms of two kinds of surface along parallel to terrace direction. Owing to terrace defect, unbinding atoms are found on surface, as fig. 3 seen, as all atoms of the column of Ga1, Ga2, N2. In Fig.3 the bond length of $\mathrm{Ga} 1$ and $\mathrm{N} 1$ increases $1.3 \%$, the bond length of $\mathrm{Ga} 2$ and $\mathrm{N} 2$ decrease $3.2 \%$, Ga-N bonds between $\mathrm{N} 1$ or $\mathrm{N} 2$ atom and the third layer Ga atom equally incline $6.1^{\circ}$ toward [ $\overline{1} 100$ ] direction comparing with primary [0001] direction. Optimized terrace height decreases $0.44 \AA$ and the width increases $0.351 \AA$, surface platform inclines $0.7^{\circ}$ toward $\left[1^{1} 00\right]$ direction, the second surface platform incline $0.6^{\circ}$ toward the opposite direction. Ga atoms on terrace surface and in the second layer platforms have all hanging bonds and electron clouds occur overlapping, however bonds are not formed.

Fig. 3 and Fig. 4 give respectively optimized geometry of [ $\left.{ }^{11 \overline{2} 0}\right]$ and [10 $\left.{ }^{10}\right]$ terrace on $\mathrm{GaN}(0001)$ surface. The same height changes are observed for surface $\mathrm{Ga}$ atoms and the second layer $\mathrm{N}$ atoms of two kinds of surface along parallel to terrace direction. Owing to the terrace defect, unbinding atoms are found on the surface. As fig. 3 shows, all atoms of the column of Ga1, Ga2, N2. In Fig.3 the bond length of Ga1 and N1 increases 1.3\%, the bond length of Ga2 and N2 decrease 3.2\%, Ga-N bonds between $\mathrm{N} 1$ or $\mathrm{N} 2$ atom and the third layer Ga atom equally incline $6.1^{\circ}$ toward $[\overline{1} 100]$ direction comparing with primary [0001] direction. Optimized terrace height decreases $0.44 \AA$, and the width increases $0.351 \AA$, while the surface platform inclines $0.7^{\circ}$ toward $\left[{ }^{1}{ }^{100}\right]$ direction, the second surface platform incline $0.6^{\circ}$ toward the opposite direction. Ga atoms found on terrace surface 
throughout the second layer platforms all have hanging bonds and electron clouds regularly overlapping, however despite these actions, bonds are not formed.

In Fig.4, atomic space and the bond length between $\mathrm{Ga}$ atoms of surface and $\mathrm{N}$ atoms of the second layer decreases respectively $84.0 \%$ and $2.2 \%$. The bond length between the second and third layer atoms increases $0.5 \%$, and incline $6.4 \%$ towards $\left[{ }^{10} 010\right]$ direction is observed. Optimized terrace height decreases $0.650 \AA$ and the width increases $0.348 \AA$, surface platform inclines $0.2^{\circ}$ toward $[1 \overline{2} 10]$ direction, terrace incline direction and angle of the second layer is similar with the surface. Our study has also exhibited proof that terrace on $\left[{ }^{11 \overline{2} 0}\right.$ ] direction has a little effect on surface atomic relaxation.
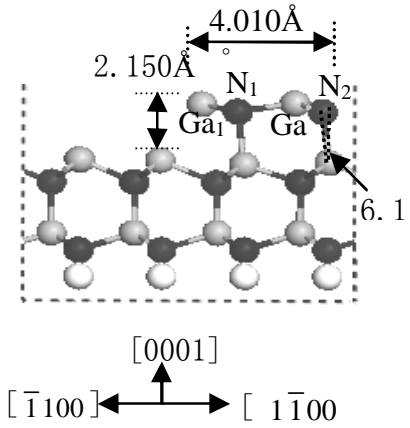

(a) Side view

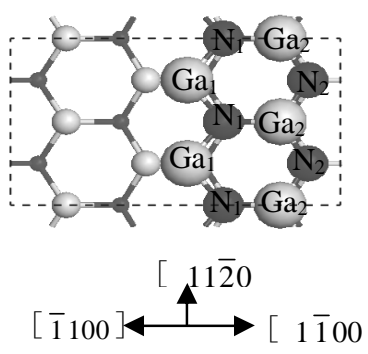

(b) Vertical view

Fig.3 Optimized geometry of terrace on $[11 \overline{2} 0]$ direction for $\mathrm{GaN}(0001)$ surface

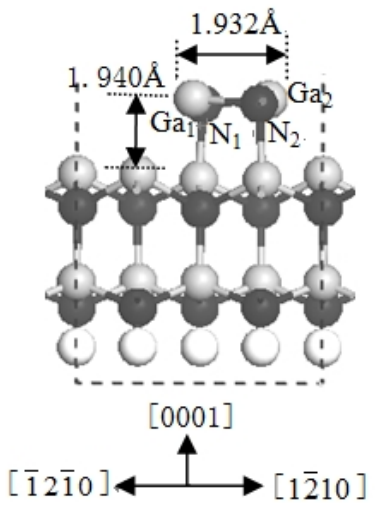

(a) Side view

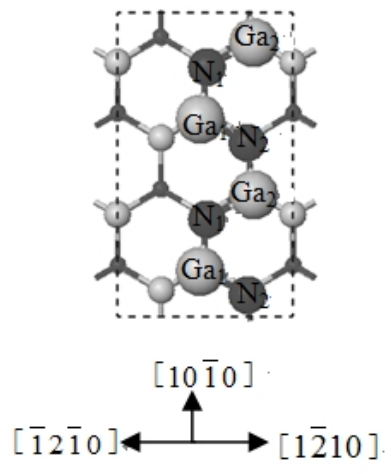

(b) Vertical view

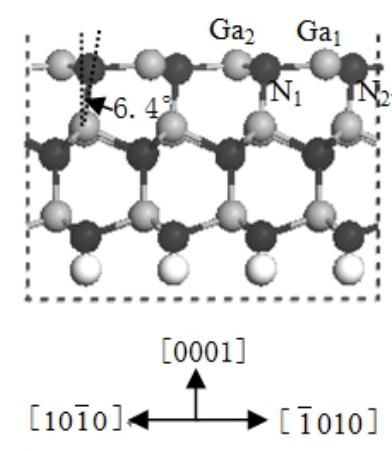

(c) Side view

Fig.4 Optimized geometry of terrace on $[10 \overline{1} 0]$ direction for $\mathrm{GaN}(0001)$ surface

Surface stability analysis: In the event surface atomic periodic arrangement is interrupted without warning, additional surface energy is created. Surface energy can reflect the object's surface's stability and can be calculated as follows ${ }^{[20-21]}$ :

$$
E_{\text {surf }}=E_{\text {tot }}^{\text {slab }}-\mu_{G a N}^{\text {bulk }} n_{N}-\mu_{G a}^{\text {bulk }}\left(n_{G a}-n_{N}\right)-\Delta \mu_{G a}\left(n_{G a}-n_{N}\right)-\mu_{H} n_{H}-E_{\text {clean }}^{\text {surf }}
$$

$E_{t o t}^{\text {slab }}$ indicates the total energy of the system, while ${ }_{\text {clean }}^{\text {surf }}$ indicates surface energy of the reference clean surface, each ${ }^{\mu_{i}}$ and $n_{i}$ their own respective indicate respectively chemical potential and atomic number of the system. $\Delta \mu_{G a}$ indicates the difference of chemical potential between element $\mathrm{Ga}$ and single crystal.

Fig.5 shows surface energy of unit area ( $\AA 2)$ of the deferent defect surface relative to clean relaxation surface of $\mathrm{GaN}(0001)$. The line defect's surface energy is directly related with the element's chemical potential; additionally, as Ga chemical potential increases, the surface energy slowly grows larger as well, which can cause surface stability to become weakened. This confirms the belief that Ga-terminated surface is relatively stable in condition of rich-nitrogen. The different defect can all 
decrease surface energy of $\mathrm{GaN}(0001)$, in other words surface stability increases, allowing the surface of terrace defect to have the better stability than line defect, terrace surface in the direction of [11 $\overline{2} 0$ ]

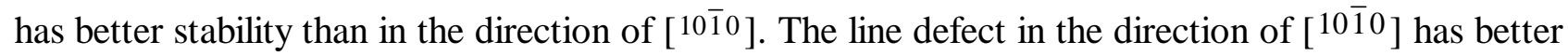
stability than in the direction of $\left[{ }^{11 \overline{2}} 0\right]$. Conclusively, when more defect surface atom relaxation is found, it is subsequently likely that the surface stability will also be poor. When line defect or terrace defect alone exist in $\mathrm{GaN}(0001)$ surface, terrace defect in the direction of [11 $\overline{2} 0$ ] and line defect in the direction of $\left[10^{\overline{1}} 0\right]$ we find the best stability, which is identical with experiment results ${ }^{[10,22]}$.

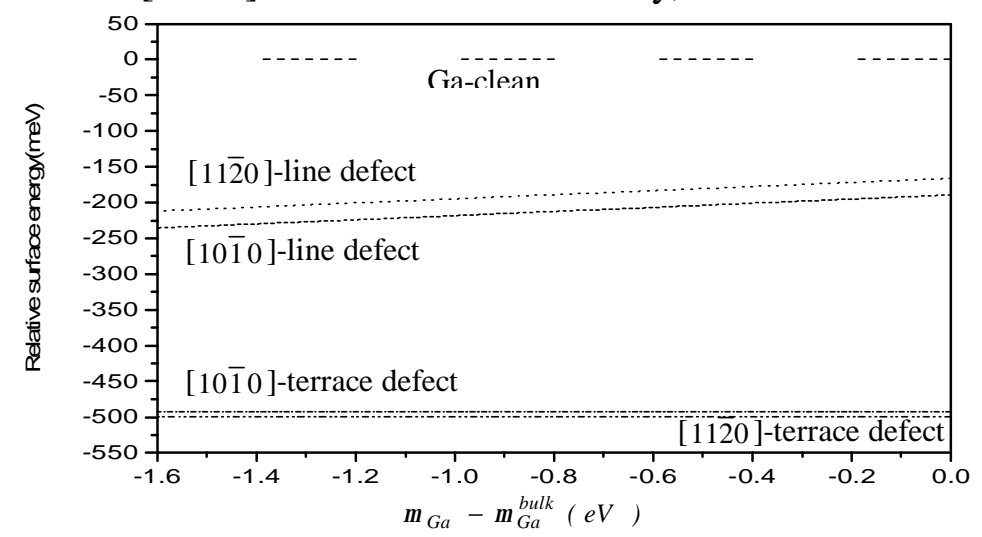

Fig.5 Surface energy of defect surface relative to clean relaxation is listed. Ga-clean indicates surface energy of clean relaxation, as zero surface energy line; $\left[{ }^{11 \overline{2} 0}\right]$-line and $\left[{ }^{10 \overline{1}} 0\right]$-line defect indicate respectively line defect surface. $\left[{ }^{11 \overline{2} 0}\right]$-terrace and $\left[{ }^{10 \overline{1}} 0\right]$-terrace defect indicate respectively terrace defect.

Analysis of the electronic structure of the surface: In attempt to perform a thorough analysis regarding the relaxation condition of atoms near defect, both the bonding electron density and Mulliken charge population of those atoms have been investigated. Table 2 lists the bonding electron density and surface properties of Ga-N on clean and defect surface. From table 2 view, the charge density of Ga1 and N1 on [ ${ }^{11} \overline{2} 0$ ] terrace surface is $0.58 \sim 0.66$ a.u. (Fig 6(c)), which is lower than 0.66 0.75 a.u. on the clean surface. Charge densities of Ga1 - N1 and Ga2 - N2 on [ ${ }^{10} \overline{1} 0$ ] terrace surface are all 0.78 0.84 a.u., which is still higher than that of the clean surface, however the terrace surface still presents metal properties ${ }^{[23]}$. The charge density around $\mathrm{Ga}$ and $\mathrm{N}$ atoms on the entire terrace surface increases, which easily combined with the foreign particles and a positive charge. Because $\mathrm{Ga}$ atoms lose electrons while $\mathrm{N}$ atoms gain electrons on the line defect surface, the binding capabilities of $\mathrm{Ga}$ and $\mathrm{N}$ atoms strengthen. As shown in Fig.6(a) and 6(b), charge density of atoms near defect are all $0.88 \sim 1.00$ a.u., particularly atoms around $\left[1{ }^{11} 0\right]$ line defect, which indicate that strong valence bond has form and hardly adsorb external particles.

Table 2 Charge density of Ga-N and surface properties of clean and defect surface

\begin{tabular}{cccccc}
\hline & Clean surface & $\begin{array}{c}{[11 \overline{2} 0] \text { Line }} \\
\text { defect surface }\end{array}$ & $\begin{array}{c}{[10 \overline{1} 0] \text { Line }} \\
\text { defect surface }\end{array}$ & $\begin{array}{c}{[11 \overline{2} 0]} \\
\text { terrace surface }\end{array}$ & $\begin{array}{c}{[10 \overline{1} 0] \text { terrac }} \\
\text { e surface }\end{array}$ \\
Ga-N charge density & $0.66 \sim 0.75$ & 1.00 & $0.88 \sim 0.97$ & $0.58 \sim 0.66$ & $0.78 \sim 0.84$ \\
Surface property & metallic bond & covalent bond & covalent bond & metallic bond & metallic bond \\
\hline
\end{tabular}




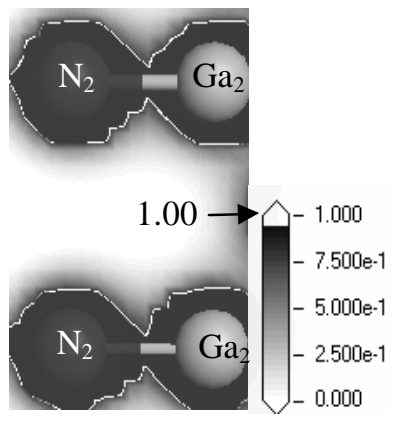

(a) Charge density on $[11 \overline{2} 0]$ line defect surface

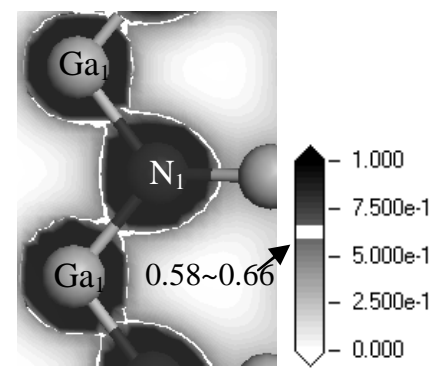

(c) Charge density on $[11 \overline{2} 0]$ terrace surface

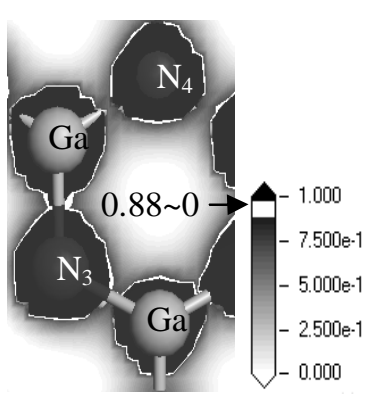

(b) Charge density on $[10 \overline{1} 0]$ line defect surface

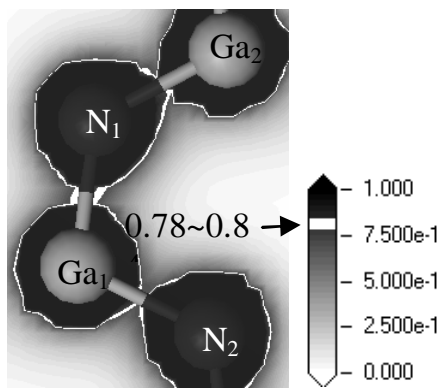

(d) Charge density on $[10 \overline{1} 0]$ terrace surface

Fig.6 Charge density (ELF) of optimized defect surface

\section{Conclusion}

Line defects on the $\left[1{ }^{11 \overline{2}}\right]$ and $[10 \overline{1} 0]$ direction and Ga-N single-layer terrace defects with $2.590 \AA$ height on Ga-terminated $\mathrm{GaN}(0001)$ surface are studied by using first-principle calculations based on density functional theory. The surface energy, charge density, and Mulliken charge population are calculated. Upon reviewing the results of the study, the final calculations show the space between $\mathrm{Ga} 3$ atom and $\left[1{ }^{11 \overline{2}} 0\right]$ line defect is the most compared with optimized clean surface; $[11 \overline{2} 0]$ line defect effecting on atoms of surface decreases with the increase of the distance; relaxation of [ $\left.{ }^{10 \overline{1}} 0\right]$ line defect has the relative regulation; the stability of defect surface in the $\left[{ }^{10} \overline{1} 0\right]$ direction is better than that in the $[11 \overline{2} 0$ ]direction. Geometry on GaN(0001) terrace surface in the $[11 \overline{2} 0]$ and $[10 \overline{1} 0]$ direction have been calculated. The results show that optimized terrace height decreases $0.44 \AA$ and the width increases $0.351 \AA$, surface platform tilt $0.7^{\circ}$ toward $\left[1^{1} 00\right.$ ] direction, the second surface platform tilt $0.6^{\circ}$ toward the opposite direction. The $\mathrm{Ga}$ atoms studied on second layer platforms of the terrace surface all have hanging bonds and electron clouds overlapping one another, however actual bonds are never formed. Terrace on the $\left[{ }^{11 \overline{2}} 0\right]$ direction has little effect on relaxation of surface atoms.

The relaxation of defect surface atoms is dependent on the actual size of the surface; the larger the surface, the lower the surface stability. In instances when line and terrace defect exists alone $\mathrm{GaN}(0001)$ surface, terrace on the $\left[11^{\overline{2}} 0\right]$ direction and line defect on the $\left[10^{\overline{1}} 0\right]$ direction is the most stable. The terrace surface platform on the $\left[{ }^{11 \overline{2} 0}\right]$ direction tilt $0.7^{\circ}$ toward $\left[{ }^{1 \overline{1} 00}\right]$ direction, as well as on the $[10 \overline{10}]$ direction tilt $0.2^{\circ}$ toward the $\left[{ }^{12} 10\right]$ direction.

As the charge density around all $\mathrm{Ga}$ and $\mathrm{N}$ atoms increases, it allows those atoms to easily combine themselves with foreign particles carrying a positive charge. $\mathrm{Ga}$ atoms lose electrons and $\mathrm{N}$ atoms get electrons on line defect surface, therefore the binding ability between Ga and $\mathrm{N}$ strengthen. The charge density of atoms near defect are all $0.88 \sim 1.00$ a.u., specifically atoms around [11 $\overline{2} 0]$ line defect, which indicate that strong valence bond has form and hardly adsorb external particles. 


\section{Acknowledgements}

This work is supported by the National Science Foundation of China (Project No.51172150) and Science and Technology Department of Sichuan Province, China; (Project No. 2014JY0091).

\section{References}

[1] G. Mula, C. Adelmann, S. Moehl, J. Oullier and B. Daudin, Phys. Rev. B. Vol.64(2001), p. 195406.

[2] B. Heying, R. Averbeck, L. F. Chen, E. Haus, H. Riechert and J. S. Speck, J. Appl. Phys., Vol.88(2000),p.1855

[3] C. Adelmann, J. Brault, G. Mula, B. Daudin, L. Lymperakis and J. Neugebauer, Phys. Rev. B, Vol.67(2003),p.165419

[4] G. Koblmüller, R. Averbeck, H. Riechert and P. Pongratz, Phys. Rev. B Vol.69(2004),p.035325

[5] H. Morkoc, S. Strite, G.B. Gao, M.E. Lin, B. Sverdlov and M. Burns, J. Appl. Phys. Vol.76(1994), 1363

[6] S.C. Jain, M. Willander, J. Narayan and R. V. Overstraeten, J. Appl. Phys.Vol.87(2000), p.965

[7] P. J. Hansen, L. Shen, Y. Wu, A. Stonas, Y. Terao, S. Heikman, D. Buttari,T. R. Taylor, S. P. DenBaars, U. K. Mishra, R. A. York, and J. S. Speck, J. Vac. Sci. Technol. B Vol. 22(2004), p.2479

[8] H. Wu, J. Yuan, T. Peng, Y. Pan, T. Han, and C. Liu, Appl. Phys. Lett.Vol. 94(2009), p.122904

[9] O. Ambacher, J. Phys. D Appl. Phys.Vol. 31(1998), p.2653

[10] W. E. Packard, J. D. Dow, K. Doverspike, R. Kaplan and R. Nicolaides, J. Mater.Res.Vol.12(1997), p.646

[11]M. A. Zaluska-Kotur, F. Krzyzewski and S. Krukowski, J. Cryst.Growth Vol.343(2012),p.138

[12] X.Q.Shen and H.Okumura, J. Cryst.Growth Vol.300(2007),p.75

[13] H. Watanabe, N. Kuroda, H. Sunakawa and A.Usui, Appl. Phys.Lett.Vol.77(2000),p.1786

[14] J. Fritsch, O. F. Sankey, K. E. Schmidt and J. B. Page, Phys.Rev. B Vol. 57(1998), p.15360

[15] M.H.Tsai,O.F.Sankey,K.E.Schmidt,and I.S.T Tsong, Mat.Sci.Eng.B, Vol.88(2002) ,p.40

[16] T.Zyweitz,J.Neigebauer and M.Scheffle, Appl.Phys.Lett. Vol.73(1998),p.487

[17] W.W. Jie, M.S. Dissertation, Chengdu:Sichuan normal university, (2010).

[18] P. Huang and C.Yang, Acta phys. Sin.Vol.60(2011), p.106801

[19] F.H.Wang, P. Krüger and J. Pollmann, Phys.Rev.B.Vol. 64(2001), 035305

[20] D.A.Drabold, S.K.Estreicher and Arno Schindlmayr, Appl.Physics.Vol.104(2007), p.165

[21] J. Wang, H. Zheng, Z.Ma, S.Prasertchoung, M.Wuttig, R.Droopad, J.Yu, K.Eisenbeiser and R. Ramesh, Appl Phys Lett.Vol.85(2004), p.2574

[22] P. J. Wellmann, S. A. Sakwe, F. Oehlschläger, V. Hoffmann, U. Zeimer and A. Knauer, J. Cryst.Growth Vol.310(2008), p.955

[23] Y. J. Du, B. K. Chang, J. J.Zhang, B. Li and X. H. Wang, Acta. Phys. Sin.,Vol. 61(2012), p.067101 